\title{
Review
}

\section{Assessment of burn depth and burn wound healing potential}

\section{Stan Monstrey ${ }^{*}$, Henk Hoeksema, Jos Verbelen, Ali Pirayesh, Phillip Blondeel}

Department of Plastic Surgery, Gent University Hospital, De Pintelaan 185, B-9000 Gent, Belgium

\section{A R T I C L E I N F O}

Article history:

Accepted 29 January 2008

Keywords:

Burns

Diagnosis

Burn depth

Healing potential

Treatment

\begin{abstract}
A B S T R A C T
The depth of a burn wound and/or its healing potential are the most important determinants of the therapeutic management and of the residual morbidity or scarring.

Traditionally, burn surgeons divide burns into superficial which heal by rapid re-epithelialization with minimal scarring and deep burns requiring surgical therapy. Clinical assessment remains the most frequent technique to measure the depth of a burn wound although this has been shown to be accurate in only $60-75 \%$ of the cases, even when carried out by an experienced burn surgeon.

In this article we review all current modalities useful to provide an objective assessment of the burn wound depth, from simple clinical evaluation to biopsy and histology and to various perfusion measurement techniques such as thermography, vital dyes, video angiography, video microscopy, and laser Doppler techniques.

The different needs according to the different diagnostic situations are considered.

It is concluded that for the initial emergency assessment, the use of telemetry and simple burn photographs are the best option, that for research purposes a wide range of different techniques can be used but that, most importantly, for the actual treatment decisions, laser Doppler imaging is the only technique that has been shown to accurately predict wound outcome with a large weight of evidence. Moreover this technique has been approved for burn depth assessment by regulatory bodies including the FDA.
\end{abstract}

C 2008 Elsevier Ltd and ISBI. All rights reserved.

\section{Contents}

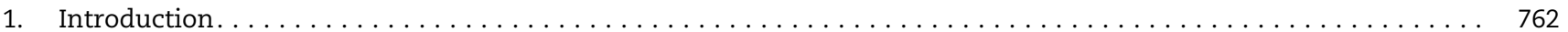

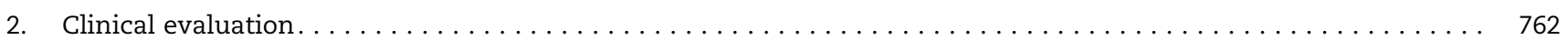

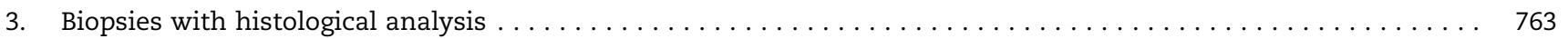

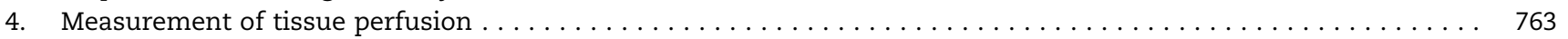

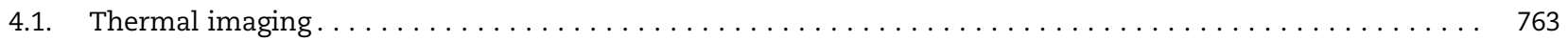

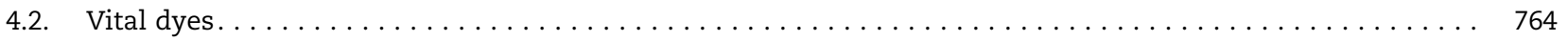

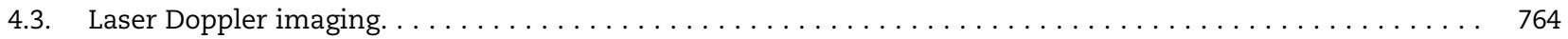

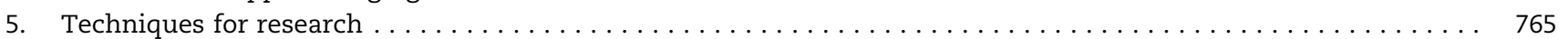

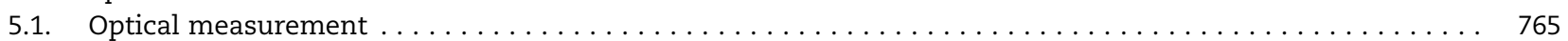

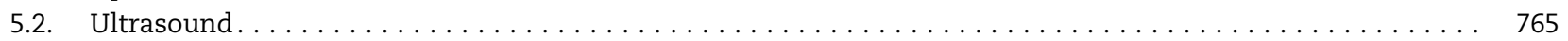

\footnotetext{
* Corresponding author. Tel.: +32 933232 27; fax: +32 93323899.

E-mail address: stan.monstrey@ugent.be (S. Monstrey). 


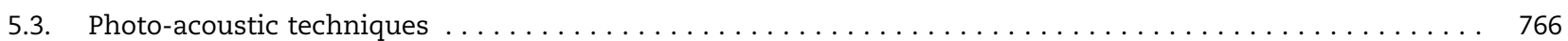

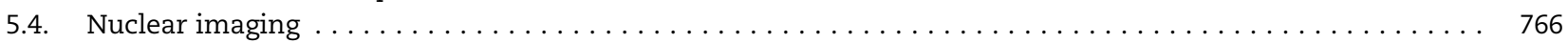

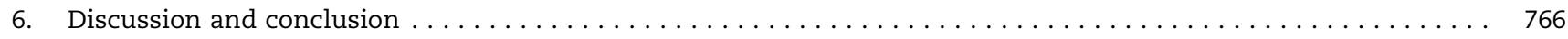

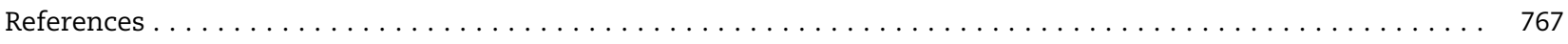

\section{Introduction}

The measurement of burn depth is an important clinical goal in the management of the burned patient. However, burn depth assessment is still an inaccurate science often governed by very subjective criteria.

The structural-anatomical classification into four categories of increasing depth of the thermal damage going from epidermal to superficial partial thickness, deep partial thickness to full thickness burns, is more of theoretical value and very difficult to determine in clinical practice [1,2]. Surgeons prefer the more clinically useful division of burns into superficial wounds healing by conservative treatment versus deep burn wound requiring surgical therapy. Unfortunately, this dichotomous classification oversimplifies reality since there never is a clear division line between the two groups.

For most burn surgeons, burn depth is better defined by the time to healing which is linked to the risk of developing hypertrophic scarring. It has been shown that if a partial thickness burn wound heals within 2 weeks, scarring is unlikely to occur, where after 3 weeks, the risk of hypertrophic scar formation is extremely high [3]. But here again a large number of burns wounds fall into the category that heals between 2 and 3 weeks and for these burns the likelihood of scarring seems to vary considerably [4]. Moreover, determination of burn depth and healing time is further complicated by the dynamic changes that have been observed during the acute post-burn period and can result in the conversion of more superficial to deep burn wound [5].

In this article we aim to review all current modalities for burn depth assessment. We considered a range of different needs according to specific diagnostic situations which can be divided in three different categories:

1. Early assessment of acute burns in the accident and emergency departments where rapid decisions are made concerning the extension and the depth of the burn which determines fluid needs, an eventual referral and the indication for escharotomy.

2. Burn depth assessment for treatment decisions of the actual burn wound as to whether or not to operate and what to excise or leave.

3. Measurement of burn depth for research purposes: when comparing different treatments, it is essential to ensure that burn depth/severity is similar in each group. This is equally important for research into wound mechanisms to help devise new treatments.

When reviewing the different techniques for burn depth assessment the following criteria must be considered:

1. Performance: precision, specificity, sensitivity and validation.
2. Ease of use: speed, mobility, patient comfort, ease of interpretation, duration, learning curve.

3. Costs involved for machine maintenance, personnel, and training.

4. The suitability for different types of burn management preferences: i.e. is it equally useful for early-excisers versus wait-and-see-ers.

\section{Clinical evaluation}

Clinical evaluation of the burn wound is the most widely used and the least expensive method of assessing burn wound depth [6]. This method relies on a subjective evaluation of the external features of the wound such as wound appearance, capillary refill, and burn wound sensibility to touch and pin prick $[1,2,7,8]$. These burn wound characteristics can be readily observed and therefore clinical assessment of the burn wound can be made immediately, easily and with minimal costs involved [1].

Unfortunately, none of the clinical features used to assess burn depth have been demonstrated to be $100 \%$ reliable and the accuracy of bedside depth assessment is widely considered to be far from optimal $[9,10]$.

With clinical judgment it is possible to diagnose very deep and very shallow burns with adequate reliability, but clinical evaluation is markedly less accurate for burns of intermediate depth which unfortunately are very common [6]. Overall estimates report that clinical depth assessment is accurate in only about $2 / 3$ of the cases [8] with the most frequent cause of error attributed to depth overestimation [11].

The second limitation of clinical assessment evolves around the validity of diagnosis [1]. There seems to be a considerable variation between burn depth assessments performed by different clinicians [12]. Not only is the base line level of experience with burn assessment variable but also the extent of tissue damage may not be immediately visually apparent as well [13]. Indeed, bedside clinical evaluation is specifically difficult in the early hours post-burn where the still viable zone of 'stasis' may denature, thereby increasing burn wound depth [5]. Recent literature has suggested that timely intervention may retard the rate and extend of such burn wound conversion [14]. Therefore an early and reliable burn depth assessment is of great importance especially at the accident and emergency department of local hospitals where burn patients often first seek attention.

For the inexperienced emergency physician, it might be useful to get expert advice, regarding early treatment or regarding a possible referral to a burn centre based on remote expert consultation using digital photographic images. This new technique has been investigated in several studies of telemetry. Roa et al. [15] noted the advantages brought about by the availability of digital photography and compared clinical diagnosis with two photographic assessments (with 
different image formats), finding similar success (90\%) with 1.5 MB BMP files and $30 \mathrm{kB}$ JPEG files as to accuracy of assessing depth from photographs. Jones et al. [16] found no difference in accuracy of assessing depth from photographs with file sizes of 2.25, 5.5, and $9 \mathrm{MB}$ per image. Use of photographs at the burn centre to aid the whole burn team has also been investigated by Nelson et al. [17]. Most staff (72\%) agreed that the usefulness extended to improved patient care: for moving patients, positioning in theatre and review of healing or complications. The disadvantages included difficulty in assessing the precise burn depth and the lack of availability out of hours. It should be stressed however, that in the very acute phase the distinction between a superficial dermal burn and a deep dermal burn is not that essential. Moreover even high resolution digital images limit three dimensionality and all digital images exist outside of the context of tactile examination [1].

More recently, the popular mobile telephone equipped with digital camera technology has been suggested by Shokrollahi et al. [18] to rapidly communicate images for remote assessment of burn area and depth. This study found high correlation between remote assessment and burns assessed live and could overcome the problem of unavailability found by Nelson et al. [17]. This was also confirmed by Dubrulle et al. [19].

\section{Biopsies with histological analysis}

Punch biopsy of burn tissue with subsequent histological analysis is frequently considered as the 'gold standard' of burn depth assessment, serving as the basis for comparison of other diagnostic modalities [6,9].

Assessment is performed by a pathologist on thin sections of the tissue following hematoxilin and eosin staining [20]. With these stains an assessment can be made of changes to cellular vitality and the denaturation caused by burns.

Burn depth is described in terms of the anatomical depth at which the boundary between healthy and necrotic tissue is observed. To improve on the precision of this technique, Watts et al. [2] used multiple assessments, computing the average of the most superficial patent blood vessels and the deepest thrombosed vessel. It has also been argued that where microvascular damage suggests a burn is partial thickness, collagen denaturation suggests it is full thickness [21].

Although biopsy with histological analysis is a well studied and widely accepted method for depth assessment, it is not without disadvantages. First of all, a biopsy is not inherently $100 \%$ accurate. Sampling error, which occurs when a nonrepresentative portion of the wound is biopsied and tissue shrinkage, which occurs when a specimen is histologically mounted, are of concern [2]. Moreover the structural damage may not necessarily correlate with functional loss, definitely not for early biopsies when considering the progressive nature of a burn wound [22]. Not only do burn wound biopsies and their microscopic examination provide only a snap shot view of the level of microvascular injury and the degree of tissue viability [22], the biopsy itself may leave an additional scar and its application may therefore be impractical in a clinical setting [23]. Employing biopsy as a depth assessment technique is also limited by the need for an experienced pathologist to interpret specimens. Finally biopsy interpretation itself is subjective from a histopathological point of view [20].

In view of the above mentioned disadvantages, the biopsy/ histology techniques continue to be excellent for experimental research and for confirmation of burn depth in wound that are treated surgically. However, the time taken to obtain results even with use of frozen sections in the OR, cannot compete with the non-invasive methods that are nowadays available. For wounds that are treated conservatively the ethics of obtaining biopsy samples is definitely questionable.

\section{Measurement of tissue perfusion}

The relationship of depth of burn and microvascular blood flow is well established [24,25].

Jackson [5] was one of the first to link some of the early clinical changes indicative of burn depth progression to the level of microvascular blood flow in the remaining dermis.

Although the patho-physiologic mechanisms responsible for burn depth progression in human burn wounds have not yet been completely elucidated, it seems that final burn depth is related to the patency of vessels in the superficial vascular plexus [2].

Because of this close relationship between burn depth and dermal blood flow, several techniques have been developed to measure the cutaneous circulation and/or tissue perfusion.

\subsection{Thermal imaging}

Thermography is based on the measurement of burn wound temperature as an indicator of their depths $[1,26]$. By exploiting the notion that deeper wounds are colder than more superficial ones because of less vascular perfusion near the wound surface, thermography is able to inversely correlate temperature with depth [27]. Watson and Vasilescu [28] found full thickness wounds to be more than $2{ }^{\circ} \mathrm{C}$ cooler than contralateral unburned skin. Still et al. [29] report that the accuracy of thermography is as high as $90 \%$ based on one degree differences in temperature at various aspects of wound. The technique has recently been revisited by Renkielska et al. [30,31] and Ruminski et al. [32]. Their novel approach was to assess temperature responses following a thermal pulse. The initial experiments in pigs found prediction of healing before or after 21 days to be statistically significant $(P<0.05)$. Although thermography is a fast imaging technique that is easy to use, it is limited by the confounding effects of ambient heat loss and sensitive timing. Evaporative loss of heat to the environment causes wounds to be interpreted as falsely deep, introducing a systematic error to this technique as was shown by Anselmo and Zawacki [33]. In addition, accuracy is compromised if wounds begin to granulate, so optimal results occur when thermography is done within 3 days of sustaining the burn [34] which precludes the use on patients transferred after day 3 after burn.

Thermal imaging is an indirect assessment of blood flow, so it is unlikely to have a role for depth assessment in burns research and it is yet to be shown clinically whether the 
limitations of thermography can be overcome by the introduction of dynamic assessments following thermal pulse stimulus. Significant improvements are needed before this technique achieves clinically significant results.

\subsection{Vital dyes}

Several nonfluorescent vital dyes such as Evans blue, patent blue $\mathrm{V}$, and bromophenol blue, have been studied for use in burn depth assessment [35]. Although these vital dyes can identify surface necrosis, they have generally been proven not to distinguish between partial and full thickness burns [29]. Given the limited amount of diagnostic information these vital dyes provide, they are regarded as having low clinical utility [6]. Fluorescent dye was first evaluated by Dingwall [36] using sodium fluorescein. Fluorescein fluorescence involves intravenous injection of fluorescein dye, followed by illumination with $360-400 \mathrm{~nm}$ ultraviolet light over burned areas. Ultraviolet light aids in depth visualization, but because it incompletely penetrates soft tissue, fluorescein fluorescence can neither differentiate between superficial and deep partial thickness burns, nor detect viable tissue that is masked by overlying escar [37,29].

Indocyanine green (ICG) is a harmless dye that is administered intravenously and then $5-10 \mathrm{~min}$ later the skin is irradiated with light at one wavelength while the fluorescence is observed at another wavelength. Presence and strength of the fluorescence indicate presence and strength of blood flow, expressed as a ratio to normal skin [38]. Recent modification of this technique using videography instead of angiography allows the capturing of dynamic changes in tissue perfusion [39]. Following an intravenous dose of ICG, laser fluorescence videography is used to create a video image of dye uptake and clearance as an indicator of tissue perfusion. Boushel et al. [40] reported that ICG video angiography determined dermal viability with high sensitivity and also had the advantage of being able to correlate structure (i.e. coagulated dermal vessels) with function (i.e. impaired local perfusion) [41]. However, Haslik et al. [42] showed that commonly used ointments and dressings had a 'massive influence' on ICG video angiography causing decreases in absorption of up to $63 \%$ leading to a dramatic overestimation of the depth of the burn wounds. Current practice standards suggest that this problem can be overcome by complete removal of all topical substances from a wound at least $10 \mathrm{~min}$ prior to ICG video angiography [42].

Although the large area and speed of imaging are significant advantages of the ICG video angiography technique, the need for injection and the limited post-injection time window could significantly detract from more widespread clinical use. Moreover ICG video angiography is also limited by the somewhat expensive and sophisticated infrastructure it requires [1].

\subsection{Laser Doppler imaging}

The laser Doppler technique has been used since 1975 for monitoring the cutaneous circulation [43]. Doppler flowmetry is based on the Doppler principles which states that when monofrequency lightwaves are reflected off moving objects, they undergo a change in frequency. By analogy, laser light that is directed at moving blood cells in sampled tissue, will exhibit a frequency change that is proportional to the amount of perfusion in the tissue [44]. In the original Doppler flowmetry a fiber optic probe in direct contact with the burn wound was used to assess microcirculation $1 \mathrm{~mm}$ below the point of probe tissue contact. Initial studies of burns with the laser Doppler technique were performed by Alsbjorn et al. [45]. Baseline measurements and measurements following local heating were made, using a contact probe and rules were derived that defined wounds of different depths that were histologically assessed as either superficial dermal, deep dermal or subdermal. An accuracy of $92 \%$ was obtained. Despite these encouraging results, the principle disadvantage remained the use of a heated probe in direct contact with one or more points of the burn surface [46-48]. Only part of the total burn surface therefore was assessed using this method with the consequent risk of an erroneous diagnosis as a result of sampling error $[46,49]$. In addition there remains the potential for pain when applying the probe to the skin surface and sepsis secondary to contamination [46]. Laser Doppler imaging (LDI) which combines laser Doppler and scanning techniques avoids the disadvantages associated with flowmetry as the whole burn may be sampled and there is no requirement for direct contact with the burn surface $[44,50]$. The accuracy of laser Doppler flowmetry and laser Doppler imaging ranges from $90 \%$ to $97 \%$ as compared to $66 \%$ with clinical evaluation only $[9,46]$. In addition the positive predictive value of the laser Doppler technique is as high as 98.4\% [51]. After scanning a burned area, LDI devices generate a color coded perfusion map that corresponds to varying burn depths. LDI is a highly valid measure for burn wound depth and its accuracy has been reported at up to $99 \%$ if infected wounds are excluded [52]. Indeed, the most recent studies addressing the critical interface between superficial and deep partial thickness burns suggest that LDI reliably predicts the level that distinguishes between burns that will or will not heal by re-epithalialisation by 3 weeks $[9,53]$. Laser Doppler imaging is the only technique that has been approved by the American Federal Drug Administration (FDA) specifically for the assessment of burns. This follows a long and consistent body of works demonstrating the efficiency of LDI in clinical studies. The technique was first used by Niazi et al. [11] who demonstrated that the accuracy of LDI assessment was $100 \%$ compared with biopsy-histology and only $65 \%$ for clinical assessment. More recently Pape et al. [9] performed an audit of their use of LDI in the assessment of burn depth and reported 97\% accuracy with LDI. These findings were supported by those of Hoeksema et al. [54] who reported LDI accuracy of $95 \%$ and $97 \%$ for LDI scans performed on day 3 and day 5 after burn, respectively compared to $52.5 \%$ and $71.4 \%$ for clinical evaluation. Jeng et al. [52] reported that use of LDI to assess burn depth enabled earlier and more objective determination of the need to excise and graft burns [55], and showed that use of LDI helped to avoid unnecessary surgery and resulted in a reduction of both costs and workload.

For paediatric patients treated surgically, Petrie et al. [56] found that the length of hospital stay fell to 9.8 days after the introduction of routine LDI assessments compared with 15.1 days before its introduction.

The LDI technique was reported as accurate for predicting burn wound healing in 57 children, average age 1 year and 10 
months [46]: for assessments made at $48 \mathrm{~h}$ the sensitivity of LDI was $90 \%$ compared with $66 \%$ for clinical evaluation; specificity was $96 \%$ for LDI compared with $71 \%$ for clinical evaluation. A further strength of this study was that cut-off levels for LDI perfusion for deep partial thickness/full thickness and superficial partial thickness burns were defined and assessments were compared with outcome at day 12 .

The strength of the LDI was further shown in a study by La Hei et al. [57] who found that its use enabled accurate burn assessments to be made in $97 \%$ of cases, without benefit of direct wound observation but with the aid of a low resolution digital color photograph (also obtained from the LDI in use).

Unlike the ICG technique, with regard to traces of creams and other wound dressings causing errors, LDI has been found to tolerate light smears of Flammazine ${ }^{\circledR}$ and remnants of Flammacerium ${ }^{\circledR}$, following procedures to avoid crust formation [58].

\section{Techniques for research}

There are many techniques that may not be suitable aids for routine clinical assessment of burns but have been shown useful for research. Some of these techniques could become clinically useful as technology improves but others, including the 'gold standard' of biopsy/histology, are never likely to attain clinical usefulness because they are restricted to single or multiple small areas and are not practical for mapping large areas of the wound.

Near infrared spectroscopic (NIRS) techniques have been assessed experimentally by Sowa et al. [59] and clinically by Cross et al. [60] in 16 patients. These spectroscopic techniques provide information on oxygen saturation and total haemoglobin when used in point mode; relative differences in oxygenation were obtained when used in the imaging mode. The single point measures were able to distinguish between superficial and full thickness burn wounds. The single point measures were able to distinguish between superficial and full thickness burn wounds.

NIRS techniques could become a promising aid in the assessment of the more important partial thickness burn wounds.

\subsection{Optical measurement}

By denaturing cellular proteins, burns alter the tissue structure and optical properties. Several novel techniques have been developed to quantify burn-induced optical variations as indicators of wound depth. Although none has been proven valid in a clinical setting, each technique is currently being studied with that end in mind.

Reflection-optical multi-spectral imaging performs a spectral analysis of reflected light from burn wounds, with the concept that necrotic tissue, scarring, and dermal vessel oxygen saturation alter absorption [61]. Reflection-optical multi-spectral imaging industrial prototypes are currently being used in various burn centers in an effort to determine clinical validity [62].

Optical coherence tomography (OCT) uses polarity measurements of birefringence amplitude orientation, and di- attenuation to assess tissue structure and function $[63,64]$. Polarization-sensitive optical coherence tomography measures the extent to which reflected light from burns has changed polarity. Reduction in collagen birefringence is thought to be related to burn depth. While polarizationsensitive optical coherence tomography has been studied in animals, it has not been demonstrated in humans [63].

The OCT technique provides an optical biopsy that could be useful in experimental situations to follow the evolution of burns and their healing but it is unlikely to have any direct impact clinically.

Orthogonal polarization spectral imaging illuminates the tissue with polarized light within the haemoglobin spectrum. It has been used for the non-invasive assessment of skin microcirculation through the surface of the human burn wound [65]. Using this technique, 2 distinct microcirculatory patterns were seen in burned skin: superficial burns had small visible dermal capillaries studded throughout the field of view, while deep burns showed large thrombosed vessels coursing a criss-crossed fashion. This disparity reflects the marked difference between the mean optical densities for superficial burns and deep burns. Orthogonal polarization spectral imaging is limited as a technique in that it requires direct tissue contact and covers only a small area in one reading.

While still in their infancy, these optical techniques offer the promise of non-invasive, non-contact, rapid assessment of burn wounds. As animal and human studies progress, these modalities may become the next innovation in burn depth diagnostics.

Video microscopy was assessed by McGill et al. [66] on patients admitted within $72 \mathrm{~h}$ after burn. Microvascular features were observed: "intact or nearly intact dermal vasculature, progressing through to large amounts of capillary destruction and haemoglobin deposition in deep partial thickness injuries and complete destruction in full thickness injuries". The findings correlated strongly with results obtained from laser Doppler imaging and clinical outcome.

Unfortunately, the field of view for video microscopy is only a few square millimetres and this probably restricts it to research applications. Moreover the requirement of perfect stability is also a limitation for clinical use.

Plasma free hemoglobin level (PFHL) has been proposed by Wong et al. [67] as a new technique for burn depth assessment. Blood samples were taken at times within the first hour after burn and examined spectrophotometrically: full thickness burns caused twice as much hemolysis as was seen from partial thickness burns and a linear correlation was found between PFHL and \%TBSA burned. PFHL peaked between 15 and 30 min after burn.

Although assessment of PFHL can be a useful technique in experimental situations to immediately confirm the depth of burns, it should be stressed that the early peak, the need for laboratory facilities and the complex nature of patient burns are likely to make this unsuitable for clinical use.

\subsection{Ultrasound}

Initial burn experiments to assess burn depth with ultrasound were performed in pulse echo mode by Goans et al. [68]. The ultrasound is reflected from skin boundaries: epidermal, 
dermal, and subcutis. By measuring the time between the echoes from each interface and assuming the speed of sound within skin is known, it is possible to calculate the thickness of each layer. Ultrasound scanning in B-mode, to give a skin cross-sectional image, was first used to assess burns by Kalus [69]. Wachtel [70] did not find the technique useful with a similar success rate to clinical assessment but in a series of experimental burns Brink et al. [71] found good correlation with histologic sections.

While traditional ultrasound requires dermal contact, noncontact ultrasound functions via a probe that is held 1 inch away from the skin [72]. Using this novel device, the operator is able to reliably distinguish epidermis, dermis, and dermal-fat interface in burned skin. With the assumption that the visualized dermal-fat interface corresponds to the depth of a full thickness dermal burn, the operator is able to estimate the depth of tissue injury with reasonable accuracy. Although not yet proven in humans, non-contact ultrasound has been demonstrated to be a rapid, accurate, non-invasive diagnostic tool in animal models.

However, as a general rule however, skin thickness varies between patients and between anatomical sites therefore knowledge of depth does not necessarily relate to knowledge of function. This assessment is aided by the identification of tissue health around sweat ducts and hair follicles which is essential for re-epithelialisation and which could be assessed by high-frequency ultrasonography [73].

This modality uses a contact probe with frequencies in the range of $20-200 \mathrm{MHz}$ to assess dermal and subdermal anatomic features. Although it requires direct skin contact, high-frequency ultrasound is able to identify dermal adnexal structures with improved resolution, thereby offering a potentially more accurate visualization of deep dermal microcirculation [73]. However, the extent to which highfrequency ultrasound can differentiate between deep dermal vasculature and edema or inflammation is not clear [74]. It has been suggested that the combination of high-frequency ultrasound with Doppler flow imaging would be a more accurate method of color flow microcirculation mapping [75]. Although high-frequency ultrasound represents a theoretical improvement upon non-contact ultrasound and standardfrequency ultrasound techniques, it is limited not only in its requirement for tissue contact but also in its as yet unproven clinical ability.

\subsection{Photo-acoustic techniques}

The principle of the photo-acoustic technique is to 'hit' the tissues with a very short pulse of light and then detect the acoustic waves from the different skin layers. Recently, this technique has been applied to experimental burn depth assessment by Sato et al. [76] using a single wavelength technique and significant differences were found between normal and superficial dermal burns and between all different burn depths. Yamazaki et al. [77] have produced tomographs, using a multi-wavelength modality, that clearly show zones of stasis. Yamazaki et al. [78] have also applied the photo-acoustic technique to the assessment of graft adhesion, attributing signal differences to neo-vascularisation.
Photo-acoustic techniques show good potential for the experimental assessment of burns but, with use restricted to small areas, they are unlikely to be applied clinically.

\subsection{Nuclear imaging}

Radio-labeled tracers have been used to map burn depth in the context of animal models. Sayman et al. [79] described the use of a ${ }^{99 \mathrm{~m}}$ Tc methoxyisonitril (MIBI) tracer to delineate areas of muscular burns in a rat model. As expected, decreased perfusion in burned tissue manifested itself as decreased presence of the radiotracer. Although highly sensitive for burn depth in this animal model, the use of radioactive tracers may add an unnecessary potential morbidity to already compromised patients. As nuclear imaging becomes more affordable and widespread, this modality may become more important as a rapid, non-invasive depth diagnosis technique.

\section{Discussion and conclusion}

Along with the extent of burn and the age of the patient, the depth of burn is a primary prognostic indicator of mortality following thermal injury. Burn depth is also the primary determinant of the patient's long term appearance and function.

Accurate assessment of burn wound depth remains an important clinical goal in the management of the acutely burned patient. Not only does depth dictate patient prognosis, it also indicates the most appropriate clinical intervention for a given wound as well. As such, understanding the relative efficacies of various modalities for evaluating burn depth, continues to be a priority.

This review has shown that burn assessment is aided by many techniques. Traditionally, there has been too much emphasis on burn depth assessment which is not entirely appropriate.

In his editorial on prognostic indicators of burns, Shakespeare [80] observed that treatment decisions are based on a prognosis of wound outcome not the diagnosis of burn injury depth. This observation reflects the practical expedient used in many studies: to assess time to healing or to assess what is healed at set time points. For this reason it is, perhaps, no longer appropriate to consider histological evaluation of biopsies as the gold standard in all studies; clinical studies that do not report histological results are not of less value, in fact those that do may be misleading because of the time and local site of sampling, methods of analysis and lack of comment functional parameters. It will remain a very important adjunct to other measures for many research studies.

Although bedside clinical evaluation remains the most widespread and least expensive method for depth diagnosis, it is accurate only about $2 / 3$ of the time and is limited by poor inter-rater reliability. Assessing burn depth in the early hours after burn is reported to be even more difficult. But for initial burn assessment at accident and emergency departments, where decisions to treat or refer need to be made, telemedicine is an effective technique to get expert advice with the simple transmission of burn images and information to a specialist 
burn centre. The most recent and effective device for this is the common mobile phone with digital photography function. Techniques are being investigated that might give an accurate burn assessment in the early hours after burn but $48 \mathrm{~h}$ after burn is usually the earliest that devices can be helpful. Therefore the opinion from a specialist burn centre, based on photographs and history, will lead to optimum decisions at this time.

For research, choice of techniques is wide and decisions must be based on the nature of the intervention being made and the observations required: clearly a sample that is removed for biopsy does not continue to function but the information gained from non-invasive techniques is unlikely to be adequate at the cellular level.

The importance of objective burn assessment for the comparison of new and different wound dressings is gaining wider acceptance. The study by Noorderbos et al. [81], comparing Trancyte dressings with silver sulphadiazine cream, was criticised by Pape and Byrne [55] because no objective selection was used to show that the 'paired' burns were of similar depth. Norbury et al. [82] suggested that Hohlfeld et al. [83] should have used LDI to assess deep dermal wounds treated with fetal skin constructs. The study by Kumar et al. [84] was the first to objectively confirm the depth of wounds selected for comparison of treatments: TranCyte, Biobrane, and silver sulphadiazine cream. The LDI assessments were essential to demonstrate that appropriate wounds were treated and that the outcomes were due to differences in the dressings and not to differences in the wounds treated. These differences could have been present if wounds had been selected on clinical grounds alone.

Most non-invasive or minimally invasive techniques for burn assessment have been initially proposed for routine use by the burns team but few have survived the test of time to become widely used. Evidence of performance is essential, particularly within the difficult category of 'indeterminate' wounds, that is adult burns that may or may not heal by day 21 (or child burns that may or may not heal by about day 14). This specific group of patients was separately considered by Heimbach et al. [8] but in other studies where clinical assessment has been compared it is as if some clinical decisions have been forced: we should follow Heimbach et al. [8] and not be afraid to admit in our publications when we simply do not know. After all, this is the main reason why many burns are treated conservatively until a clear opinion can be formed. The performance of any technique for burns of clinically indeterminate depth defines much of its usefulness.

Where two techniques have similar accuracies, ease of use is most important: e.g. ICG requires intravenous injections and although imaging is fast, all burn sites must be imaged within a short time window (5-10 min). Burn assessments are most efficiently performed at the dressing change so patient comfort and nursing techniques are of greatest importance: in a multi site burn it is frequently preferred to remove dressings sequentially and this would not suit ICG fluorescence imaging whereas there are no such time limits for the LDI technique. Speed and area of imaging are important, particularly with infants and young children. In this respect LDI has disadvantage because images currently take about one minute per scan. Sedation is commonly used during dressing changes and Holland et al. [46] have reported good LDI results for child burns.

Over the last 14 years one technique, LDI, has gained the confidence of many surgeons to aid their treatment decision making. Accuracy of assessment with LDI is frequently reported at about 95\% and the 'precision' of the assessment has recently been extended to classification of burns into 3 groups: those healing within 14 days, healing between 14 and 21 days and burns predicted not to heal within 21 days. The predictions are made between $48 \mathrm{~h}$ and 5 days post-burn. LDI is the only technique that has been approved by regulatory bodies, including the FDA, for burn assessment. Its use has been shown to reduce the surgical workload $[55,56]$ by avoiding unnecessary operations and earlier surgery for appropriate cases; a goal that has been the aim of all burn assessment techniques beforehand. No doubt other techniques will eventually supersede LDI but for now it is the best available choice.

Over 20 years ago Heimbach et al. [8] posed the question, 'Burn depth estimation-man or machine?'. This review has shown that there are several techniques are capable of assessing burn depth or, more often, predicting the healing time, commonly grouping cases into either more/less than 14 days or more/less than 21 days. The simple question of 'man OR machine?' suggests to much polarisation and we should consider the important contributions that can be made by both man AND machine combined: wound preparation, infection, burn site, and other considerations will remain important to treatment decisions; ensuring that measurement conditions are appropriate to the technique used and interpretation of technical information are also essential manual inputs for the accurate assessment of burn prognosis.

Until novel modalities unfold in the future, a combination of clinical evaluation and another modality - LDI being the most favourable option - is currently advocated for optimal delineation of the depth of acute burn wounds and for prognosis and treatment guidance.

\section{Conflict of interest statement}

None of the authors has any financial interest whatsoever in any of the techniques or instruments mentioned in this article.

\section{R E F E R E N C E S}

[1] Devgan L, Bhat S, Aylward B, Spence S. Modalities for the assessment of burn wound depth. J Burns \& Wounds 2006;5:7-15.

[2] Watts A, Tyler M, Perry M, Roberts A, McGrouther D. Burn depth and its histological measurement. Burns 2001;27: 154-60.

[3] Bombaro K, Engrav L, Carrougher G, Wiechamn S, Faucher $\mathrm{L}$, Costa $\mathrm{B}$, et al. What is the prevalence of hypertrophic scarring following burns? Burns 2003;29:299-302.

[4] Cubison T, Pape S, Parkhouse N. Evidence for the link between healing time and the development of hypertrophic scars (HTS) in paediatric burns due to scald. Burns 2006;32:992-9. 
[5] Jackson D. The diagnosis of the depth of burning. Br J Surg 1953;40:588-96.

[6] Heimbach D, Engrav L, Grube B, Marvin J. Burn depth: a review. World J Surg 1992;16:10-5.

[7] Godina M, Derganc M, Brcic A. The reliability of clinical assessment of the depth of burns. Burns 1978;4:92-6.

[8] Heimbach D, Afromowitz M, Engrav L, Marvin J, Perry B. Burn depth estimation-man or machine. J Trauma 1984;24:373-8.

[9] Pape S, Skouras C, Byrne P. An audit of the use of laser Doppler imaging (LDI) in the assessment of burns of intermediate depth. Burns 2001;27:233-9.

[10] Droog E, Steenbergen W, Sjöberg F. Measurement of depth of burns by laser Doppler perfusion imaging. Burns 2001;27:561-8.

[11] Niazi Z, Essex T, Papini R, Scott D, Mclean N, Black M. New laser Doppler scanner, a valuable adjunct in burn depth assessment. Burns 1993;19:485-9.

[12] Milseki W, Atiles L, Purdue G, Kagan R, Saffle J, Herndon D, et al. Serial measurements increase the accuracy of laser Doppler assessment of burns wound. J Burn Care Rehabil 2003;24:187-91.

[13] Johnson R, Richard R. Partial-thickness burns: identification and management. Adv Skin Wound Care 2003;16:178-87.

[14] Kao C, Garner W. Acute burns. J Plast Reconstr Surg 2000;105:2482-92.

[15] Roa L, Gómez-Cía T, Acha B, Serrano C. Digital imaging in remote diagnosis of burns. Burns 1999;25:617-23.

[16] Jones O, Wildon D, Andrews S. The reliability of digital images when used to assess burn wound. J Telemed Telecare 2003;9(Suppl 1):S22-4.

[17] Nelson L, Boyle M, Taggart I, Watson S. Are burns photographs useful. Burns 2006;32:876-9.

[18] Shokrollahi K, Sayed M, Dickson W, Potokar T. Mobile phones for the assessment of burns: we have the technology. Burns 2007;24:753-5.

[19] Dubrulle F, Blondeel Ph, Roche N, Van Landuyt K, Hamdi M, Monstrey S. Cell phone imaging for teleconsulting in plastic surgery. Astractbook Joint Meeting Belgian and Dutch Societies of Plast Surg 2006;15.

[20] Kahn A, McCrady V, Rosen V. Burn wound biopsy. Multiple uses in patient management. Scand J Plast Surg 1979;13: 53-6.

[21] Chvapil M, Speer D, Owen J, Chvapil T. Identification of the depth of burn injury by collagen stainability. Plast Reconstr Surg 1984;73:438-41.

[22] Robson M, Kucan J, Paik K, Eriksson E. Prevention of dermal ischemia after thermal injury. Arch Surg 1978;113:621-5.

[23] Saranto J, Rubayi S, Zawacki B. Blisters, cooling, antithromboxanes and healing in experimental zone of stasis burns. J Trauma 1983;23:927-33.

[24] Boykin J, Eriksson E, Pittman R. In vivo microcirculation of a scald burn and the progression of postburn dermal ischemia. Plast Reconstr Surg 1980;66:191-8.

[25] Zawacki B. Reversal of capillary stasis and prevention of necrosis in burns. Ann Surg 1974;180:98-102.

[26] Mladick R, Georgiade N, Thorne F. A clinical evaluation of the use of thermography in determining degree of burn injury. Plast Reconstr Surg 1966;38:512-8.

[27] Lawson R, Gaston J. Temperature measurements of localized pathological processes. Ann NY Acad Sci 1964;121:90-8.

[28] Watson A, Vasilescu C. Thermography in plastic surgery. J R Coll Surg Edinb 1972;17(4):247-52.

[29] Still J, Law E, Klavuhn K, Island T, Holtz J. Diagnosis of burn depth using laser-induced indocyanine green fluorescence: a preliminary clinical trial. Burns 2001;27:364-71.

[30] Renkielska A, Nowakowski A, Kaczmarek M, Dobke M, Grudzinski J, Karmolinski A, et al. Static thermography revised-and adjunct method for determining the depth of the burn injury. Burns 2005;31:768-75.

[31] Renkielska A, Nowakowski A, Kaczmarek M, Ruminski J. Burn depth evaluation based on active dynamic IR thermal imaging-a preliminary study. Burns 2006;32:867-75.

[32] Ruminski J, Kaczmarek M, Renkielska A, Nowakowski A. Thermal parametric imaging in the evaluation of skin burn depth. IEEE Trans Biomed Eng 2007;54:303-12.

[33] Anselmo V, Zawacki B. Infra-red photography as a diagnostic tool for the burn ward. Proc Soc Photo-Optical Instr Eng 1973;8:181.

[34] Liddington M, Shakespeare P. Timing of the thermographic assessment of burns. Burns 1996;22:26-8.

[35] Atiyeh B, Gunn S, Hayek S. State of the art in burn treatment. World J Surg 2005;29:131-48.

[36] Dingwall J. A clinical test for differentiating second from third degree burns. Ann Surg 1943;118:427.

[37] Gatti J, LaRossa D, Silverman D, Hartford D. Evaluation of the burn wound with perfusion fluorometry. J Trauma 1983;23:202-6.

[38] Green H, Bua D, Anderson R, Nishioka N. Burn depth estimation using indocyanine green fluorescence. Arch Dermatol 1992;128:43-9.

[39] Kamoloz L, Andel H, Haslik W, Donner A, Winter W, Meiss] $\mathrm{G}$, et al. Indocyanine green video andiographies help to identify burns requiring operation. Burns 2003;29:785-91.

[40] Boushel R, Langberg H, Olesen J, Nowak M, Simonsen L, Bülow J, et al. Regional blood flow during exercise in humans measured by near-infrared spectroscopy and indocyanine green. J Appl Physiol 2000;89:1868-78.

[41] Sheridan R, Schomaker K, Lucchina L, Hurley J, Yin L, Tompkins R, Jerath. Burn depth estimation using indocyanine green fluorescence : initial human trial. J Burn Care Rehabil 1995;16:602-4.

[42] Haslik W, Kamolz L, Andel H, Winter W, Meissl G, Frey M. The influence of dressings and ointments on the qualitative and quantitative evaluation of burn wounds by ICG video-angiography: an experimental setup. Burns 2004;30:232-5.

[43] Stern M. In vivo evaluation of the micro-circulation in the leg by coherent light scattering. Nature 1975;254:56-8.

[44] Essex T, Byrne P. A laser Doppler scanner for imaging blood flow in skin. J Biomed Eng 1991;13:189-94.

[45] Alsbjorn B, Micheels J, Sorensen B. Laser Doppler flowmetry measurements of superficial dermal, deep dermal and subdermal burns. Scand J Plast Reconstr Surg 1984;18:75-9.

[46] Holland A, Martin H, Cass D. Laser Doppler imaging prediction of burn wound outcome in children. Burns 2002;28:11-7.

[47] Micheels J, Alsbjorn B, Sorensen B. Laser Doppler flowmetry: a new non-invasive measurement of microcirculation in intensive care? Resuscitation 1984;12:31-9.

[48] Atiles L, Mileski W, Spann K, Purdue G, Hunt J, Baxter C. Early assessment of pediatric burn wounds by laser Doppler flowmetry. J Burn Care Rehabil 1995;16:596-601.

[49] Shakespeare PG. Looking at burn wound: the A.B. Wallace Memorial Lecture 1991. Burns 1992;18:287-95.

[50] Brown R, Rice P, Bennet N. The use of laser Doppler imaging as an aid in clinical decision making in the treatment of vesicant burns. Burns 1998;24:692-8.

[51] O’Reilly T, Spence R, Taylor R, Scheulen J. Laser Doppler flowmetry evaluation of burn wound depth. J Burn Care Rehabil 1989;10:1-6.

[52] Jeng J, Bridgeman A, Shivnan L, et al. Laser Doppler imaging determines need for excision and grafting in advance of clinical judgement : a prospective blinded trial. Burns 2003;29:665-70.

[53] Monstrey S, Hoeksema H, Spence R, Jeng J, Wilson D, Pape S. Clinical assessment of burn wound healing potential by 
laser Doppler Imaging (LDI): results of a prospective, multi centre study. In: Abstract Book 38th Annual Meeting Am. Burn Assoc.. 2006. p. 4-7.

[54] Hoeksema H., Vandesijpe K., Hamdi M., Van Landuyt K., Blondeel Ph., Monstrey S. Accuracy of early burn depth assessment by laser Doppler Imaging on different days postburn. Burns 2008: submitted for publication.

[55] Pape S, Byrne P. Safety and efficacy of TransCyte for the treatment of partial-thickness burns. J Burn Care Rehabil 2000;21:390.

[56] Petrie N, Norbury W, Fogarty B, Philp B, Barrat J, Dziewulski $P$. The use of laser Doppler imaging to reduce operative intervention in the treatment of paediatric burns. In: Abstract Book 12th Congress of the International Society for Burn Injuries (ISBI); 2004.

[57] La Hei E, Holland A, Martin H. Laser Doppler imaging of paediatric burns: burn wound outcome can be predicted independent of clinical examination. Burns 2006;33:550-3.

[58] Ng D, Tay S, Booth S, Gilbert Ph, Dheansa B. The use of laser Doppler imaging for burn depth assessment after application of Flammacerium. Burns 2007;33:396-7.

[59] Sowa M, Leonardi L, Payette J, Fish J, Mantsch H. Near infrared spectroscopic assessment of hemodynamic changes in the early post-burn period. Burns 2001;27:214-49.

[60] Cross K, Leonardi L, Payette J, Gomez M, Levasseur M, Schattka B, et al. Clinical utilization of near-infrared spectroscopy devices for burn depth assessment. Wound Repair Regen 2007;15:332-40.

[61] Anselmo V, Zawacki B. Multispectral photographic analysis. A new quantitative tool to assist in the early diagnosis of thermal burn depth. Ann Biomed Eng 1977;5:179-93.

[62] Shah S, Bachrach N, Spear S, Letbetter D, Stone R, Dhir R, et al. Cutaneous wound analysis using hyperspectral imaging. Biotechniques 2003;34:408-13.

[63] Park B, Saxer C, Srinivas S, Nelson J, de Boer J. In vivo burn depth determination by high-speed fiber-based polarization sensitive optical coherence tomography. J Biomed Opt 2001;6:474-9.

[64] Jiao S, Yu W, Stoica G, Wang L. Contrast mechanisms in polarization-sensitive Mueller-matrix optical coherence tomography and application in burn imaging. Appl Opt 2003;42:5191-7.

[65] Milner S, Bhat S, Gulati S, Gherardini G, Smith C, Bick R. Observations on the microcirculation of the human burn wound using orthogonal polarization spectral imaging. Burns 2005;31:316-9.

[66] McGill D, Sorensen K, Mackay I, Taggart I, Watson S. Assessment of burn depth: a prospective, blinded comparison of laser, Doppler imaging and videomicroscopy. Burns 2007;33:833-42.

[67] Wong C, Song C, Heng K, Kee I, Tien S, Kumarasinghe P, et al. Tan K Plasma free haemoglobin: a novel diagnostic test for assessment of the depth of burn injury. Plast Reconstr Surg 2006;117:1206-13.

[68] Goans R, Cantrell Jr J, Meyers F. Ultrasonic pulse-echo determination of thermal injury in deep dermal burns. Med Phys 1977;4:259-63.

[69] Kalus A, Aindow J, Cauldfield M. Application of ultrasound in assessing burn depth. Lancet 1979;27:188-9.

[70] Wachtel T, Leopold G, Frank H, Frank D. B-mode ultrasonic echo determination of depth of thermal injury. Burns Inc Therm Inj 1986;12:432-7.

[71] Brink J, Sheets P, Dines K, Etchison M, Hanke C, Sadove A. Quantitative assessment of burn injury in porcine skin with high-frequency ultrasonic imaging. Invest Radiol 1986;21:645-51.

[72] Iraniha S, Cinat M, Vanderkam V, Boyko A, Lee D, Jones J, et al. Determination of burn depth with noncontact ultrasonography. J Burn Care Rehabil 2000;21:333-8.

[73] Foster F, Zhang M, Zhou Y, Liu G, Mehi J, Cherin E, et al. Starkoski. A new ultrasound instrument for in vivo microimaging of mice. Ultrasound Med Biol 2002;28:1165-72.

[74] Goertz D, Christopher D, Yu J, Kerbel R, Burns P, Foster F. High-frequency color flow imaging of the microcirculation. Ultrasound Med Biol 2000;26:63-71.

[75] Goertz D, Yu J, Kerbel R, Burns P, Foster F. High-frequency 3-D color-flow imaging of the microcirculation. Ultrasound Med Biol 2003;29:39-51.

[76] Sato S, Yamazaki M, Saitoh D, Tsuda H, Okada Y, Obara M, et al. Photo-acoustic diagnosis of burns in rats. J Trauma 2005;59:1450-6.

[77] Yamazaki M, Sato S, Ashida H, Saito D, Okada Y, Obara M. Measurement of burn depths in rats using multiwavelength photo-acoustic depth profiling. J Biomed Opt 2005;10(6):064011.

[78] Yamazaki M, Sato S, Saitoh D, Okada Y, Ashida H, Obara M. Photo-acoustic monitoring of neovascularities in grafted skin. Lasers Surg Med 2006;38:235-9.

[79] Sayman H, Demir M, Cetinkale O, Ayan F, Onsel C. A method to evaluate microcirculatory vascular patency of full thickness burn in an animal model. Panminerva Med 1999;41:5-9.

[80] Shakespeare P. Prognostic indicators and burns. Burns 2003;29:105-6.

[81] Noorderbos J, Doré C, Hansbrough J. Saftely and efficacy of Transcyte for the treatment of partial-thickness burns. J Burn Care Rehabil 1999;20:275-81.

[82] Norbury W, Jeschke M, Herndon D. Tissue engineered fetal skin constructs for pediatric burns. Crit Care 2005;9:533-4.

[83] Hohlfeld J, de Buys Roessingh A, Hirt-Burri N, Chaubert P, Gerber S, Scaletta C, et al. Tissue engineered fetal skin constructs for paediatric burns. Lancet 2005;366:840-2.

[84] Kumar R, Kimble R, Boots R, Pegg S. Treatment of partialthickness burns: a prospective, randomized trial using Transcyte $^{\text {TM }}$. ANZ J Surg 2004;74:622-6. 